SLAC-PUB-8283

Oct, 1999

\title{
Search for Free Fractional Electric Charge Elementary Particles
}

\author{
V. Halyo, P. Kim, E. R. Lee, I. T. Lee, D. Loomba, and M. L. Perl円 \\ Stanford Linear Accelerator Center \\ Stanford University, Stanford, California 94309 USA
}

\begin{abstract}
We have carried out a direct search in bulk matter for free fractional electric charge elementary particles using the largest mass single sample ever studied - about $17.4 \mathrm{mg}$ of silicone oil. The search used an improved and highly automated Millikan oil drop technique. No evidence for fractional charge particles was found. The concentration of particles with fractional charge more than 0.16 e (e being the magnitude of the electron charge) from the nearest integer charge is less than $4.71 \times 10^{-22}$ particles per nucleon with $95 \%$ confidence.
\end{abstract}

\footnotetext{
${ }^{1}$ Work supported by the Department of Energy, contract DE-AC03-76SF00515.
} 
Direct observation of free fractional charge elementary particles would be an undisputed signature of physics beyond the Standard Model. In this paper we present the results of an improved Millikan oil drop experiment designed to look for such particles. The apparatus made it possible to generate and measure the charges of multiple columns of multiple drops simultaneously, each drop being $7.6-11.0 \mu \mathrm{m}$ in diameter. This allowed us to have a large throughput of $4.17 \times 10^{7}$ drops or about $17.4 \mathrm{mg}$, of silicone oil.

In the Standard Model there are no fractional charge color singlet particles. However such particles are expected in physics beyond the Standard Model such as superstring theory. In heterotic superstring models there can be either gauge coupling unification with color singlet fractional charge particles or no fractional charge particles but also no unification. In fact all superstring models built to date have unification at the price of introducing fractional charge particles [1]. Other models for fractional charge particles are outlined in [2]. There is no reason for fractional electric charge elementary particles to be necessarily excluded. Our motivation for these bulk matter searches is the possibility that these particles may have been produced in the early universe and some abundance remains today.

There are however no confirmed discoveries of free particles with fractional electric charge. Searches have been made using accelerators, cosmic rays [3] and in bulk matter [ [4], [5]. Searches in bulk matter fall into two classes: those that attempt to concentrate the fractional charge particles before the search [6] and those that directly search through all of a bulk matter sample [7], [8]. Our preference is for direct bulk matter searches because it is frequently difficult to make a reliable estimate of the efficiency of the pre-concentration method. Our previous search using about $1 \mathrm{mg}$ of silicone oil set an upper limit of less than $4.76 \times 10^{-21}$ particles per nucleon [B] . The largest mass sample previously used in a direct bulk matter search was $4.9 \mathrm{mg}$ of niobium [9] again with a negative result.

In our experiment, drops are ejected through a silicon micromachined orifice and fall through air under the influence of gravity and an alternating vertical electric field. The drops are imaged by a digital charge coupled device (CCD) camera interfaced to a computer. The same computer is used to simultaneously collect and analyze the data and to monitor and control the experiment.

The drop generators we used consist of a glass fluid reservoir tube with a micromachined silicon orifice plate having $7-10 \mu \mathrm{m}$ hole diameter which is thermally welded to the end of the tube [10], [11]. A piezoelectric transducer disk made from lead zirconate titanate is attached to the lower portion of the tube. The dropper is filled with $5 \mathrm{cS}$ silicone oil. Silicone oil was chosen because it has low vapor pressure and the right viscosity to generate stable drops. Drop ejection is initiated by an electrical pulse that causes the piezoelectric transducer disk to contract radially on the glass, forcing a drop to form. The diameter of the drops can be varied by a factor of two by adjusting the pulse height and duration, using the method described in [11]. Once the parameters are set, the drop diameter remains constant to better than $1 \%$.

The drops are generated at $4 \mathrm{~Hz}$ producing two columns separated by $300 \mu \mathrm{m}$. Once the drops are produced they fall into an electric field produced by a parallel plate capacitor 
formed by an upper square ground plate of dimension $10 \mathrm{~cm} \times 10 \mathrm{~cm}$ and a lower round high voltage plate $7.62 \mathrm{~cm}$ in diameter. The plates are placed horizontally $0.81 \mathrm{~cm}$ apart with rectangular slits of dimension $1.27 \mathrm{~cm} \times 0.08 \mathrm{~cm}$ to allow the passage of multiple columns of drops. The chamber and optical components are mounted on a vibrationally damped optical table. The electric field plates and the dropper are contained within two layers of transparent polycarbonate shielding since the drops are sensitive to convection due to their small radius.

The drops are backlit by red LEDs strobed at $10 \mathrm{~Hz}$ with a $56 \mu$ s pulse width. The light is diffused by a ground glass screen to create uniform illumination. A $135 \mathrm{~mm}$ focal length lens $18 \mathrm{~cm}$ away from the dropper focuses the image of the drops onto the CCD camera. The camera is used to image the positions of the falling drops. The active region of the CCD is $6.4 \mathrm{~mm} \times 4.8 \mathrm{~mm}(736 \times 242$ pixels $)$ where the $6.4 \mathrm{~mm}$ edge is chosen along the trajectory of the falling drops to maximize the number of position measurements and to avoid image distortion caused by camera interlacing. The optical system has a magnification of 2.7 so that the actual field of view of the falling drops is $2.37 \mathrm{~mm}$ vertically and $1.77 \mathrm{~mm}$ horizontally. A high speed video framegrabber captures images from the CCD camera for computer analysis. The drops have an average terminal velocity of $1.3-3.2 \mathrm{~mm} / \mathrm{s}$ depending on the drop radius so that each drop is in the field of view for 8-11 sequential images. In order to ensure the control of the experiment and to help rule out fractional charge artifacts, temperature, manometer pressure, vibration and humidity are monitored.

To find the positions of the drops in an image, the analysis program first applies a brightness level threshold to isolate the relevant pixels. The pixels which are above the threshold are used to calculate an approximate optical center (centroid) for each drop. A high accuracy calculation is then done by using the 20 darkest pixels in a $10 \times 10$ pixel window around the approximate centroid to calculate a precise centroid, where each pixel is weighted by its intensity after subtracting the background value. Once the centroid positions of each image have been measured, the sequence of centroids corresponding to the trajectory of each drop is extracted from the stream of data by a tracking algorithm. The core of the algorithm examines several consecutive images and considers all possible combinations of centroids. The combination which form physically consistent trajectories are grouped to form the initial trajectory of a drop. Once an initial trajectory has been found, it is possible to predict the position of that drop in future images. If a centroid is found in the predicted position, it is associated with the appropriate drop. When a drop left the field of view it is passed to the analysis code. The software is capable of online operation at high rates, and is not currently a limiting factor in this experiment. The search has three data sets; in chronological order Set I consisted of $1.4 \mathrm{mg}$ of $7.6 \mu \mathrm{m}$ average diameter drops, Set II consisted of $10.1 \mathrm{mg}$ of $10.4 \mu \mathrm{m}$ average diameter drops, and Set III consisted of $5.9 \mathrm{mg}$ of $9.4 \mu \mathrm{m}$ drops. These data sets with different drop diameters helped us to verify that we understood our charge measurement process.

To understand how drop charge and mass are measured consider a drop falling under the influence of gravity in the presence of a vertical electric field that alternates between two discrete states, up and down. Since the drop falls in air, it reaches a terminal velocity. The 
two equations that govern the motion of the drops are given by Stoke's law:

$$
\begin{aligned}
& m g+E_{\downarrow} Q=6 \pi \eta r v_{\downarrow} \\
& m g-E_{\uparrow} Q=6 \pi \eta r v_{\uparrow}
\end{aligned}
$$

where $m$ is the drop mass, $Q$ is the drop charge, $r$ is the drop radius, $\eta$ is the viscosity of air and $v_{\downarrow}, v_{\uparrow}$ are the measured terminal velocities of the drops for the two directions of the electric field, $E_{\downarrow}, E_{\uparrow}$. We define $v_{e}$ and $v_{g}$ to be

$$
v_{e}=\frac{\left(v_{\downarrow}-v_{\uparrow}\right)}{2} \quad v_{g}=\frac{\left(v_{\downarrow}+v_{\uparrow}\right)}{2}
$$

We know the mass of the drop since the density of silicone oil is known $\left(\rho_{\text {oil }}=913.0 \mathrm{~kg} / \mathrm{m}^{3}\right)$ and we measure the radius using

$$
r=3 \sqrt{\frac{\eta}{2 g\left(\rho_{\text {oil }}-\rho_{\text {air }}\right)}} \sqrt{v_{g}}
$$

Using the measured velocities we calculate the charge of the drop in units of the electron charge e

$$
q \equiv \frac{Q}{e}=C v_{e} \sqrt{v_{g}}
$$

where $\mathrm{C}$ is

$$
C=\frac{18 \pi}{e} \sqrt{\frac{2}{\left(\rho_{\text {oil }}-\rho_{\text {air }}\right) g}} \frac{1}{E_{\downarrow}+E_{\uparrow}} \eta^{3 / 2}
$$

The charge of the drop is calculated by finding the best fit to the sequence of centroid position measurements. In addition a variety of different physical effects had to be corrected to achieve the best required charge accuracy.

Two rectangular slits in the center of the electric field plates, which allow passage of the drops, cause a spatial nonuniformity in the electric field. This nonuniformity combined with the induced dipole on the drop produce small changes in the apparent terminal velocity. This dipole force monotonically reduce the velocity of the drops as the drops fell. By measuring the gradient in the electric field we were able to calculate that this effect was $3.15 \%$ of $v_{g}$.

There is also an aerodynamic effect on the trajectory of the drops. The air in the vicinity of the columns of falling drops is dragged downwards changing the apparent terminal velocity of the drops. The resulting steady flow of the air causes the drops to reach maximum velocity halfway between the plates and then decelerate. The magnitude of the effect was $1.46 \%$ of $v_{g}$.

Since we have done our measurement $0.2 \mathrm{~mm}$ higher than halfway between the plates, the two effects acted on the drops simultaneously with opposite sign which led to a change of $1.69 \%$ of $v_{g}$. We chose the center of our operating region to be where the two effects maximally cancel each other. In the analysis, these two phenomena are corrected simultaneously by fitting the velocities of the drops to a second order polynomial. 
There is an additional interesting aerodynamic effect. Since we had an imbalance in the number of positively and negatively charge drops, there was a net motion of the drops, and hence of the air, which oscillated with the alternating electric field. This caused a shift in the measured charge on the order of $0.1 \mathrm{e}$ which was corrected.

After applying the above corrections we require that all drops used in the data sample meet the measurement criteria listed in Table [1. The first cut removes drops with charges higher than 4.5 since the measurement accuracy decreases with charge. The second cut removes drops with less than 6 centroids in order to have at least two charge measurements per drop. The third cut checks the consistency of the charge within a drop and the last cut checks for drops with high residual to eliminate tracking artifacts.

\begin{tabular}{|cc|}
\hline \hline cuts & Percentage removed \\
\hline$|q|<4.5$ & $3.056 \%$ \\
$N>6$ & $0.215 \%$ \\
$\delta q<0.2$ & $0.342 \%$ \\
$\mathrm{R}<8 \sigma_{v}$ & $0.0399 \%$ \\
\hline \hline
\end{tabular}

Table 1: The drops used in the data sample were subjected to cuts on the charge $q(|q|<$ 4.5 e), on the number of centroids $N(N>6)$, consistency of charge measurements of one drop $\delta q(\delta q<0.2 \mathrm{e})$, and the residuals $R\left(R<8 \sigma_{v}\right)$, where $\sigma_{v}$ is the uncertainty in the velocity due to Brownian motion. Percentages removed by each cut are in order of application.

These criteria removed $3.653 \%$ of the total drops. Figure 1 shows the data after applying the last three cuts described above, specifically $4.14 \times 10^{7}$ drops. We see sharp peaks at integer numbers of charges and no drops with charges further than 0.14 e from the nearest integer charge, other than a single drop at $q=0.294$.

Figure 2 shows the residual charge distribution of $q_{c}$, which is defined as $q_{c} \equiv q-N_{c}$ where $N_{c}$ is the signed integer closest to $q$, for data Set II. It displays a superposition of integer charge peaks centered at zero. The peaks at each integer charge have a Gaussian distribution shape. The standard deviation $\left(\sigma_{q}\right)$ at charge zero is $0.018 \mathrm{e}$; higher charges result in a larger charge measurement error since $\sigma_{q}$ gets contribution from terms involving $v_{e} / v_{g}$. Table 2 lists the contributions to $\sigma_{q}^{2}$; the contributions are from Brownian motion, centroid measurement and the electric field non-uniformity between the plates.

The search for drops with fractional charge is clarified in Figure 0 by the superposition of all data sets using the variable $q_{s} \equiv|q|-N_{s}$, where $N_{s}$ is defined to be the largest integer less than $|q|$. This is the entire data remaining after the application of the cuts. There is no background subtraction. Again one sees at $q_{s}=0.294 \mathrm{e}$, the sole drop charge measurement that lies outside of the integer tails. We have applied the following experimental philosophy to this measurement. In searching for a rare phenomenon it is important to apply the same data selection criteria to all the data as we have done. The drop with $q=0.294$ fits all of 


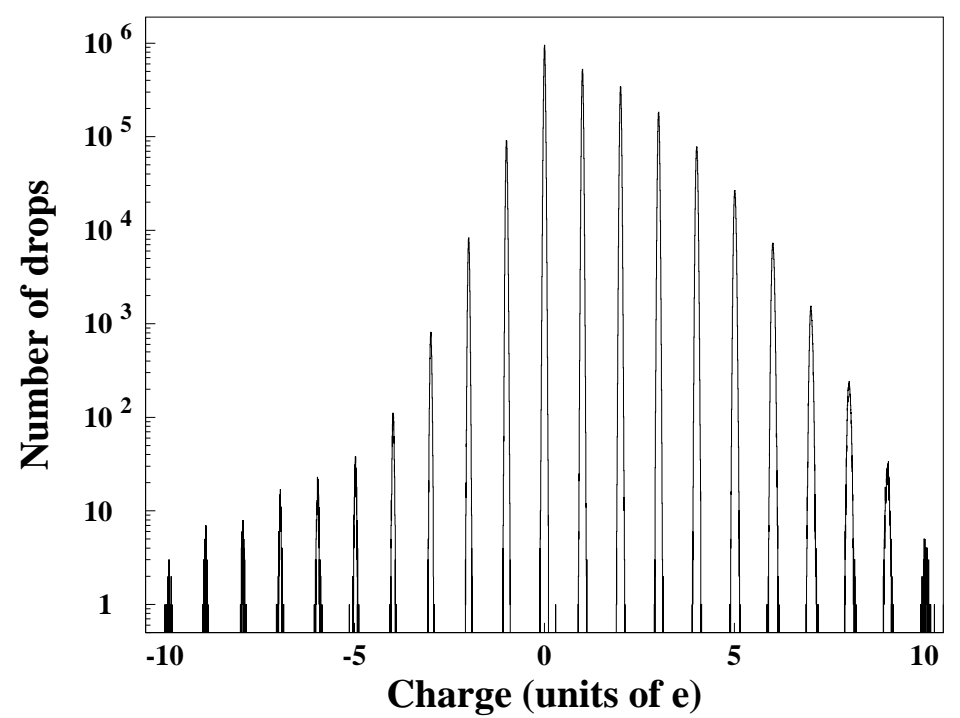

Figure 1: The charge distribution of $4.14 \times 10^{7}$ drops.

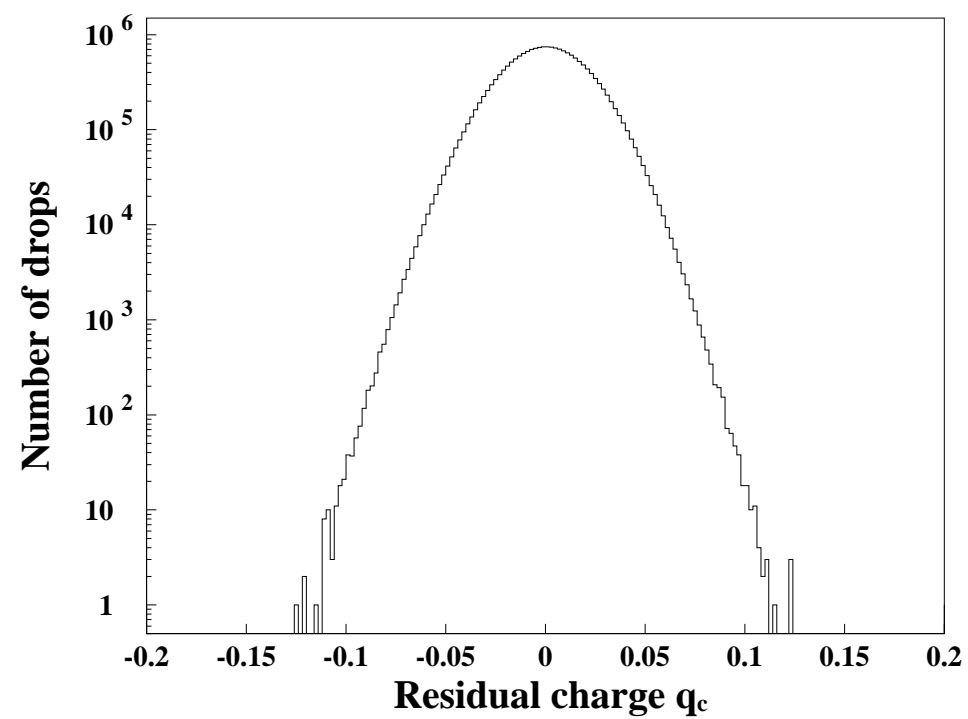

Figure 2: The residual charge based on $1.885 \times 10^{7}$ drops. The residual charge is defined as $q_{c}=q-N_{c}$. 


\begin{tabular}{|cccc|}
\hline \hline Source of Error & Set I & Set II & Set III \\
\hline Brownian motion & $57.0 \%$ & $40.9 \%$ & $42.1 \%$ \\
Centroid measurement errors & $36.2 \%$ & $47.8 \%$ & $48.2 \%$ \\
Electric field non-uniformity & $6.8 \%$ & $11.4 \%$ & $9.7 \%$ \\
\hline \hline
\end{tabular}

Table 2: The contribution to the charge measurement error $\sigma_{q}^{2}$ for each data.

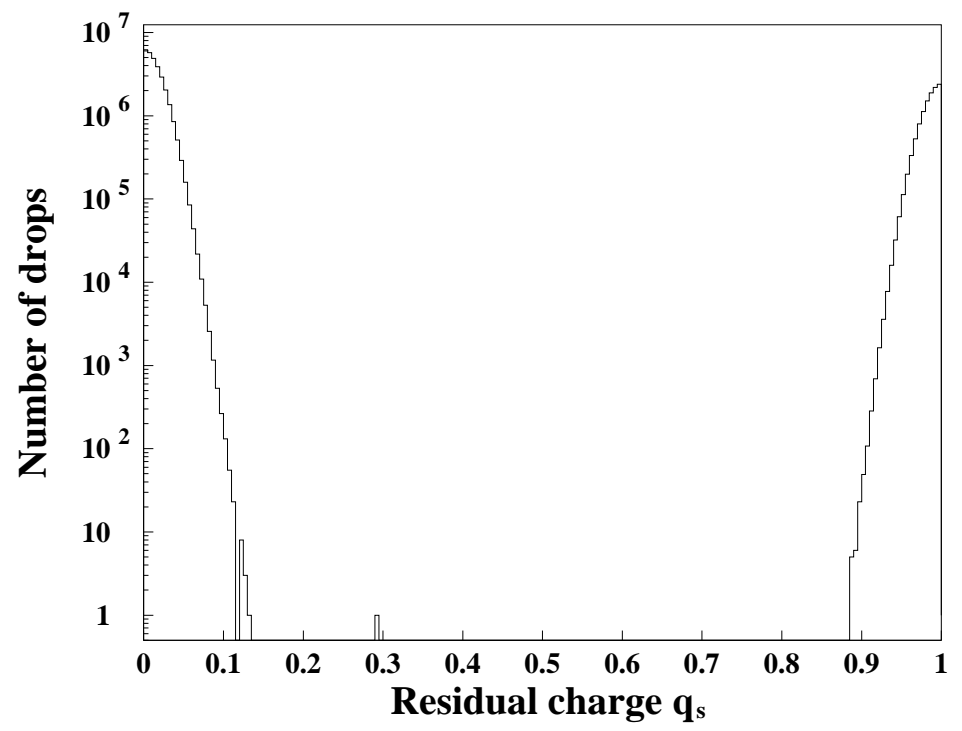

Figure 3: The residual charge on all the data. The residual charge $q_{s}$ is defined $q_{s}=q-N_{s}$.

our criteria and we do not know if it is the first indication for some background that begins to appear at the 1 in $4 \times 10^{7}$ level or if it has more significance. Our only choice is to repeat the experiment with a larger sample and we intend to do so.

Table 3 presents $95 \%$ confidence upper limits on the number of fractional charge particles per nucleon in silicone oil for each data set. We set conservative limits by counting the number of events in the signal region defined as within $2 \sigma$ of each fractional charge, and calculating Poisson limits without background subtraction. Figure 4 shows the combined $95 \%$ confidence upper limits on the number of fractional charge particles per nucleon in silicon oil for the entire run. We did not find any evidence for free fractional charge particles. We found with $95 \%$ confidence that in silicone oil the concentration of particles with fractional charge more than 0.16 e from the nearest integer charge is less than $4.71 \times 10^{-22}$ particles per nucleon except in the region $0.26-0.34$ e where the upper limit is $2.98 \times 10^{-22}$ particles per nucleon.

We have demonstrated several advantages of our Millikan method compared to the levitometer method [9] for searching for fractional charge particles in bulk matter. The Millikan method allows a broad charge range to be studied with good charge resolution and it provides 


\begin{tabular}{|ccccc|}
\hline \hline Data set & $\mathrm{D}(\mu \mathrm{m})$ & Mass $(\mathrm{mg})$ & Range & Upper limit \\
\hline I & 7.6 & 1.35 & $0.15-0.26$ & $3.84 \times 10^{-21}$ \\
& & & $0.34-0.84$ & $3.84 \times 10^{-21}$ \\
& & & $0.26-0.34$ & $6.07 \times 10^{-21}$ \\
II & 10.4 & 10.13 & $0.16-0.84$ & $5.12 \times 10^{-22}$ \\
III & 9.4 & 5.92 & $0.17-0.86$ & $8.76 \times 10^{-22}$ \\
& & & & \\
Total & & 17.4 & $0.17-0.26$ & $2.98 \times 10^{-22}$ \\
& & & $0.34-0.84$ & $2.98 \times 10^{-22}$ \\
& & & $0.26-0.34$ & $4.71 \times 10^{-22}$ \\
\hline \hline
\end{tabular}

Table 3: Final result from the three runs of the experiment including the combined limit on the total mass examined, drop diameter D, sample mass, the range of residual $q_{c}$, the $95 \%$ CL upper limit on the density of fractional charge particles per nucleon.

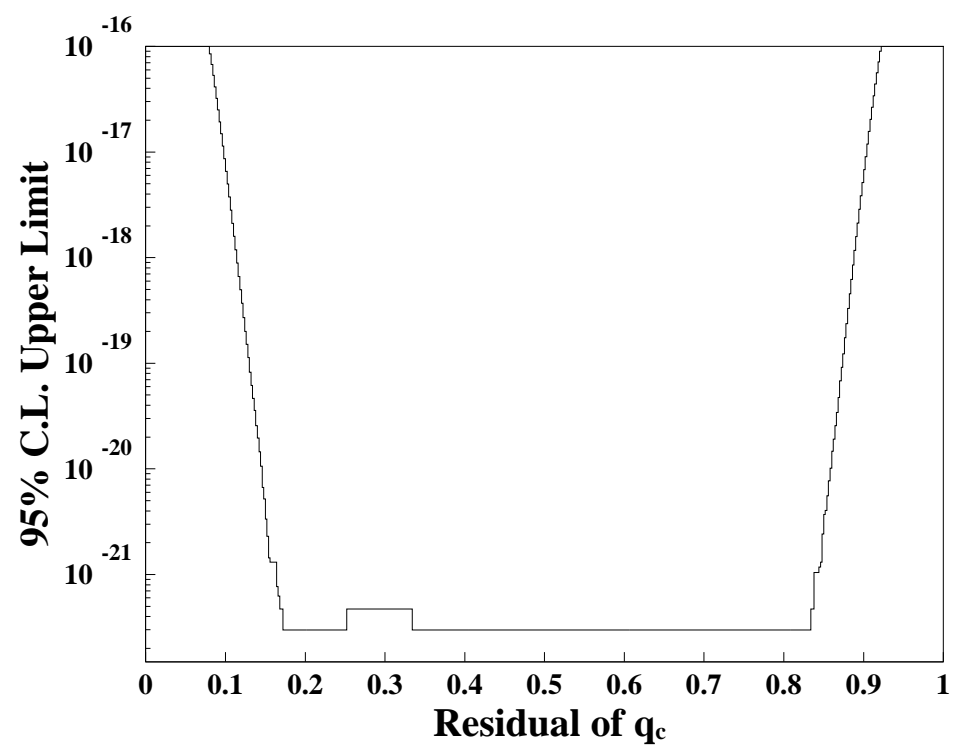

Figure 4: The $95 \%$ C.L. upper limit on density of fractional charge particles per nucleon vs. residual of $q_{c}$. 
natural self-calibration of the charge measurement. It is amenable to automation and simple replication and it permits a relatively large amount of material to be examined. There is no obvious limit to the amount of material to be studied [12].

Searches in bulk refined matter such as silicone oil, niobium, or iron suffer from the uncertainty of whether a fractional charge particle would remain in the material during the chemical or physical refining process [2], [13]. Pure material also suffer from the uncertainty of whether the geochemical and geophysical processes that concentrate a mineral in a local region of the Earth's crust would also carry along any elementary fractional charge particles. Therefore, there is great value in searching in unprocessed and unrefined bulk matter such as meteorites and certain primordial terrestrial minerals. Our subsequent searches for fractional charge particles will use drops containing such materials. 


\section{References}

[1] A. N. Schellekens, Phys. Lett. B 237, 363 (1990).

[2] M. L. Perl and E. R. Lee, Am. Journ. of Phys. 65, 698 (1997).

[3] L. Lyons, Phys. Rep. 129, 225 (1985)

[4] P.F. Smith, Ann. Rev. Nucl. Part. Sci. 39, 73-111 (1989).

[5] L. Jones, Rev. Mod. Phys. 49, 717 (1977).

[6] D. C. Joyce et al., Phys. Rev. Lett. 51, 731 (1983).

[7] P. F. Smith et al., Phys. Lett. B 171, 129 (1986).

[8] N. M. Mar et al., Phys. Rev. D 53, 11 (1986). Results from other major bulk matter searches are also listed.

[9] P. F. Smith et al., Phys. Lett. B 153, 188 (1985).

[10] E. R. Lee and M. L. Perl, U.S. Patent 5943075 (1999).

[11] E. R. Lee et al., to be published.

[12] D. Loomba et al., submitted to Rev. Scientific Instruments.

[13] M. L. Perl, Phys. Rev. D 57, 7 (1988). 